\title{
A New Electromagnetic Powder Deposition System
}

By:

J.L. Bacon

D.G. Davis

R.J. Pollizzi

R.L. Sledge

J.R. Uglum

R.C. Zowarka

United Thermal Spray Conference, Sept. 15-18, 1997, Indianapolis Indiana

ASM International

PR - 231

Center for Electromechanics

The University of Texas at Austin

PRC, Mail Code R7000

Austin, TX 78712

(512) $471-4496$ 


\title{
A New Electromagnetics Powder Deposition System
}

\author{
J.L. Bacon, D.G. Davis, R.J. Polizzi, R.L. Sledge, J.R. Uglum, and R.C. Zowarka \\ Center for Electromechanics \\ The University of Texas at Austin \\ Austin, Texas
}

\begin{abstract}
Existing state of the art thermal spray processes (HVOF, DGun, Plasma Spraying) are limited to powder velocities of about $1 \mathrm{~km} / \mathrm{sec}$ because they rely on the thermodynamic expansion of gases. A new thermal spray process using electromagnetic forces can accelerate powder particles to a final velocity of up to $2 \mathrm{~km} / \mathrm{sec}$. At this velocity powder particles have sufficient kinetic energy to melt their own mass and an equivalent substrate mass on impact.

The process is based on railgun technology developed by the Department of Defense. A railgun is filled with argon gas and a high energy electrical pulse, provided by a capacitor bank, drives the gas down the railgun to a final velocity of up to $4 \mathrm{~km} / \mathrm{sec}$. This gas passes over a powder cloud and accelerates the powder through drag forces. The electrical and powder discharge frequency can be adjusted so that the deposition rate and thermal input to the substrate can be controlled.
\end{abstract}

THE ELECTROMAGNETIC POWDER DEPOSITION (EPD) system is a viable alternative to existing build-up methods and is based on proven "railgun" technology. Railguns have been under development for 15 years by the DoD and are high energy capacity electrical systems designed for rapid acceleration of an object. Railguns are effective in accelerating projectiles to high velocities exceeding $2 \mathrm{~km} / \mathrm{sec}$. Current thermal spray technology limits coating velocities to the $1 \mathrm{~km} / \mathrm{sec}$ range. Figure $1 \mathrm{com}-$ pares several thermal spray processes in terms of particle velocity and gas temperature and shows how EPD is predicted to perform. The data for the other processes was obtained from a paper describing a "Cold Gas-Dynamic Method" [1]. In the EPD system, equipment similar to a railgun accelerates the coating material.

The acceleration capability of electromagnetic systems is expected to produce coatings with superior density and bond strength properties. In most existing thermal spray systems the deposited material must impact the substrate as a liquid. In the EPD system a powder particle can impact the substrate in a solid state and then transition to a liquid state. A velocity of $2 \mathrm{~km} / \mathrm{sec}$ provides sufficient energy to melt a metal powder particle and an equal amount of substrate mass upon impact. This interaction provides the potential for forming a fusion bond between the deposited material and the substrate.

The EPD system sprays powder in a series of discrete discharges much like a D-Gun. The powder is accelerated by a high velocity column of argon gas. The gas column is accelerated using a railgun powered by a capacitor bank. The bank is used to produce a very short duration, high magnitude electrical pulse (a peak current magnitude of $160 \mathrm{kA}$ and $120 \mu \mathrm{sec}$ duration) which is conducted by a segment of argon plasma called the armature. As current increases, energy is deposited in the argon plasma increasing its tem-

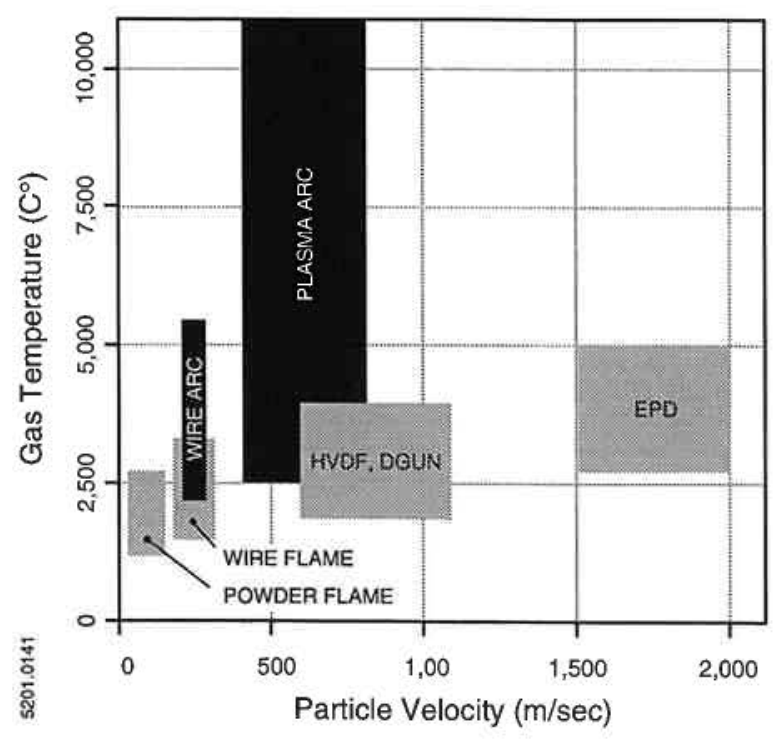

Figure 1. A comparison of particle velocity and gas temperature for several thermal spray processes. 
perature. Current moving through the armature generates an electromagnetic Lorentz force which acts on the plasma, accelerating it down the length of the railgun. Figure 2 shows a simple railgun and the electrical currents and magnetic fields which interact to create the Lorentz force (the cross product of the current density vector and the magnetic field vector). As the armature moves down the railgun, it pushes a column of gas in front of it. The gas column is shock compressed so that its density and length continues to increase with time. Powder is accelerated by viscous drag forces generated by the gas column.

The system under development will provide 10 discharges at a $30 \mathrm{~Hz}$ rate. In August 1997, coatings will be made and evaluated to determine the viability of the system. The system is expandable to 100 discharges at the rate of 30 $\mathrm{Hz}$ with the present power supply concept. After successfully obtaining coatings a new power supply will be developed for continuous duty operation.

\section{EPD Laboratory Experiment}

Process Description. Figure 3 shows the basic elements of the EPD system. A pulse forming network (PFN), which is a capacitor bank designed to provide a specific current profile, provides electrical energy to a square bore accelerator (SBA). The railgun is filled with argon and near its breech is a Radio Frequency (RF) arc initiator. Powder is fed into the last $1 / 3$ of the SBA through two injection ports. When the Triggered Vacuum Switch (TVS) is closed the PFN discharges into the SBA, driving a plasma armature down the length of the SBA. The plasma armature pushes a column of argon gas in front of it at supersonic speed. The shocked column of gas increases in length as more and more argon gas is swept up. This process of pushing a shocked column of gas is referred to as snowplowing [2]. The shocked column of argon gas passes over the injected powder and accelerates the powder to a high velocity by viscous drag force. The snowplow action has been simulated and experimentally verified [3].

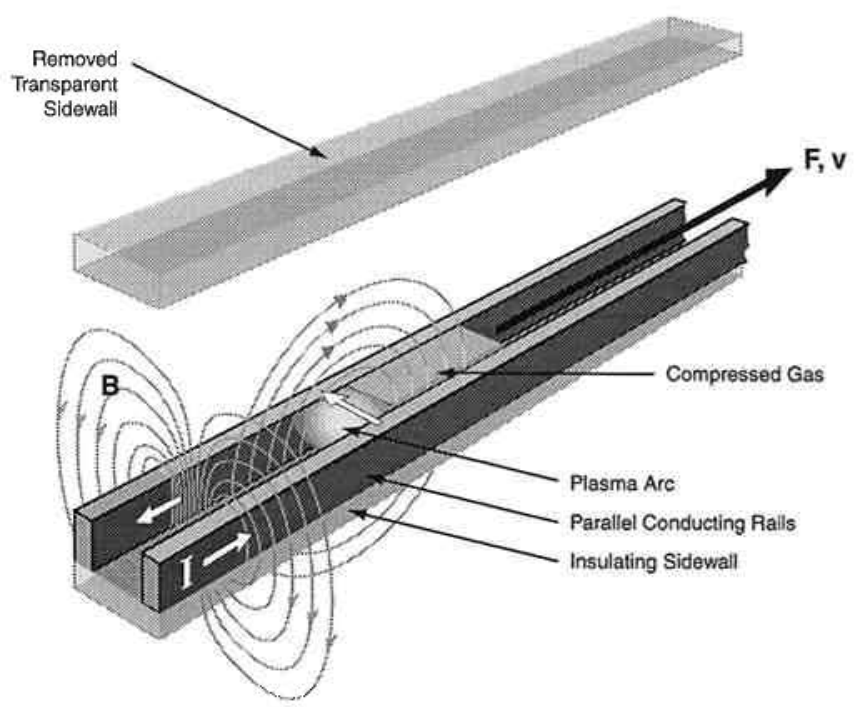

Figure 2. The plasma armature is accelerated down the length of the railgun by an electromagnetic Lorentz force generated by the by the interaction of the magnetic fields surrounding the rails and the current flowing through the armature.

Once the PFN has discharged, the TVS opens. The PFN must then be recharged by the recharge capacitor. This is accomplished in a two step process. First, the Insulated Gate Bipolar Transistor (IGBT) switch is closed and current flow is initiated through the charging inductor. The current through the inductor builds to a predetermined level such that the inductor stores sufficient electrical energy to recharge the PFN. Next, the Silicon Controlled Rectifier (SCR) switch is closed and the IGBT switch is opened. The current flowing through the inductor is diverted into the PFN, and the PFN is recharged. The SCR switch passively opens when the charging current goes from positive to negative. The TVS switch is closed and the cycle is repeated.

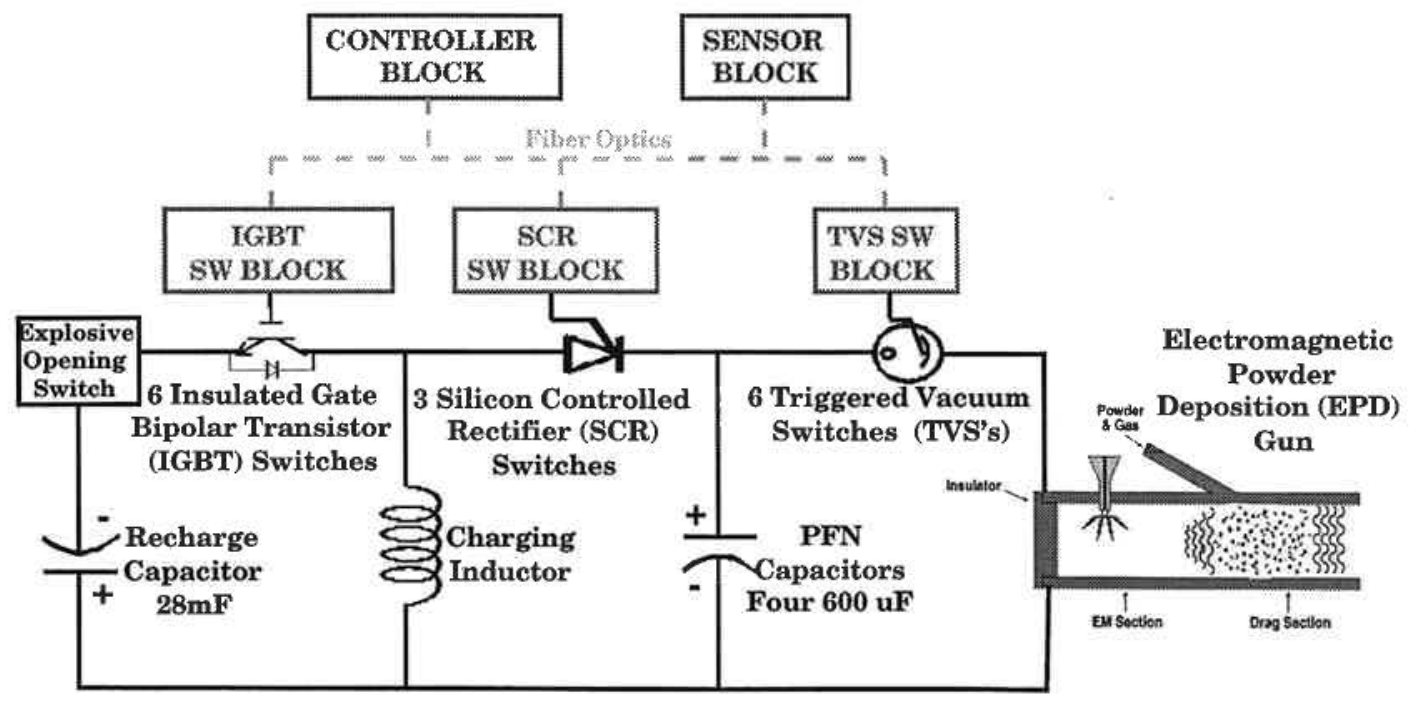

Figure 3. The basic elements of the EPD system. 


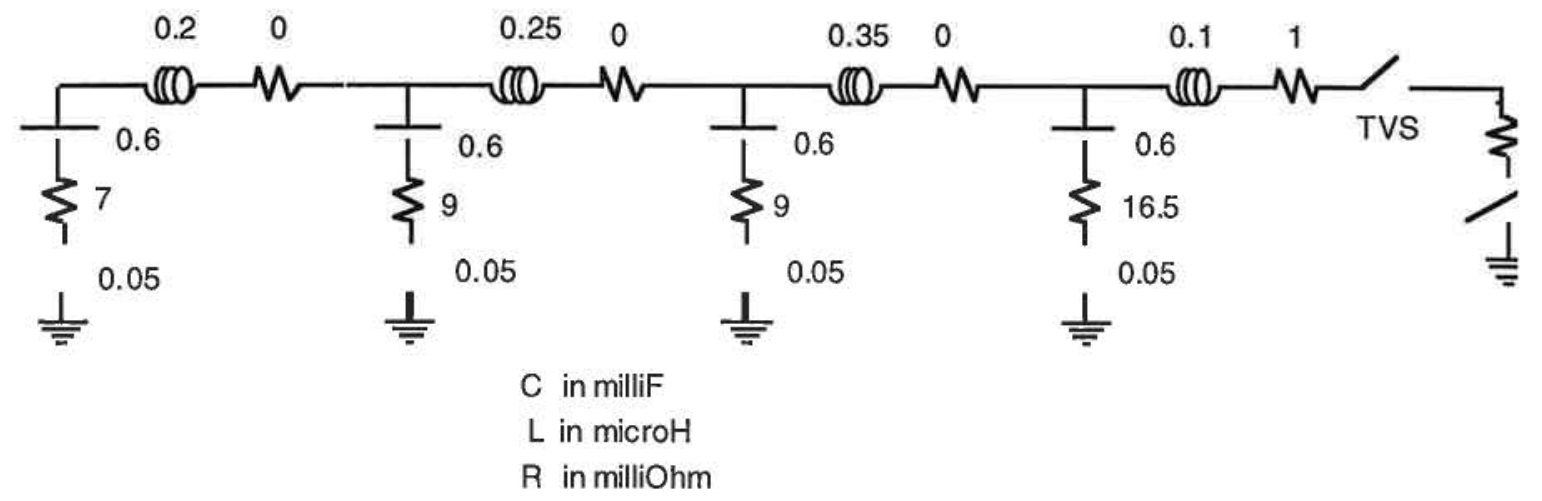

Figure 4. An electrical schematic of the Pulse Forming Network (PFN).

The IGBT switch, the SCR switch and the TVS switch each have their own control switch blocks. The switch blocks provide gate signals when commanded by the controller block. Each block is galvanicly isolated and communicated with only through fiber optics. This insures that the switch blocks receive no stray signals generated by the high electromagnetic fields present during the charging and discharging cycles. A sensor block monitors the health of the system and shuts the system down if a fault is detected. The worst case would be if the IGBT switch didn't open and the six high powered IGBTs were exposed to excessive current. To prevent this, the sensor block looks for an over current condition and can send a signal to the explosive opening switch causing it to explode, which physically opens the circuit. This is a safety feature which hopefully will be rarely used during the operation of the system.

\section{System Description.}

Square Bore Accelerator. The SBA accelerates a plasma armature through the interaction of the magnetic field produced by current flowing through the rails and the current flowing through the armature. The force acting on the armature is given by:

$$
\mathrm{F}=\frac{1}{2} \mathrm{~L}^{\prime} \mathrm{T}^{2}
$$

where $\mathrm{F}=$ accelerating force applied to the armature

$\mathrm{L}^{\prime}=$ the inductance gradient

I $=$ the current

It is advantageous to keep the current magnitude as low as possible to limit the amount of rail heating and ablation. A high inductance gradient is desirable and is dependent on rail geometry. A square bore was chosen to maximize the inductance gradient and to provide a more stable plasma armature. Equation (1) was derived from the Lorentz equation:

$$
\mathrm{F}=\mathrm{J} \times \mathrm{B}
$$

where $\mathrm{J}=$ current density

$$
\mathrm{B}=\text { magnetic flux density }
$$

The SBA is filled with argon gas ported in at the RF arc initiator and a shield gas manifold at the muzzle. The plasma armature is formed at the RF arc initiator when the PFN is discharged into the SBA. The plasma armature reaches its peak velocity quickly and snowplows an argon gas column down the length of the railgun and through powder particles which are injected into the last $1 / 3$ of the SBA, a region referred to as the drift zone. This is an electrically inactive region of the gun, i.e. the current pulse ends when the plasma armature reaches the drift zone. The flow rate of the powder is adjusted so that the drift zone is just filled with the argon gas and powder mixture before the next discharge.

Pulse Forming Network. To avoid plasma instabilities [3] the PFN must provide current to the SBA with a rapid rise time (less than $25 \mu \mathrm{sec}$ ) and the entire pulse must be very short (about $125 \mu \mathrm{sec}$ ). The ideal current waveform would jump instantly to $150 \mathrm{kA}$, remain constant for about $100 \mu \mathrm{sec}$ and drop instantly to $0 \mathrm{kA}$. Figure 4 shows the 4 stage PFN designed to meet these requirements. Figure 5 shows the current waveform generated by a finite difference computer code used to model the PFN.

The simulations indicate that the plasma armature reaches peak velocity early in the current pulse (less than 40 $\mu \mathrm{sec})$. The remaining pulse provides energy to continue snowplowing additional argon gas which is swept up into the shocked gas column. The momentum of the shocked gas column is proportional to the area under the current squared.

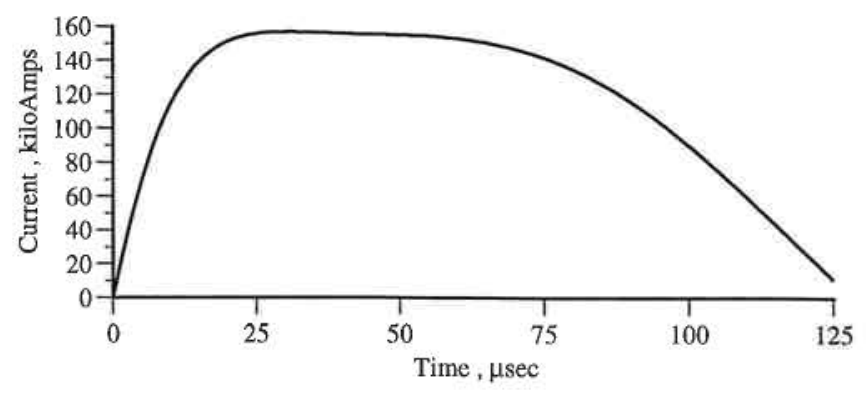

Figure 5. Gun current generated by the PFN circuit. 
The PFN, which has an equivalent resistance of $26 \mathrm{~m} \Omega$, is not well matched to the railgun load, which has an impedance of 3 to $4 \mathrm{~m} \Omega$. Most of the PFN's energy is deposited thermally into the damping resistors. Alternative power supplies which are more efficient are discussed in the Future Production Type section of this paper.

Recharge Bank. The PFN stores enough energy for one discharge. After the PFN discharges it is recharged by the recharge bank in a sequence described in the EPD Laboratory Experiment - Process Description section of this paper. Initially, one recharge bank module will be fabricated. This module has enough energy to provide ten discharges. Additional shot capability can be added by building a second recharge module which would be electrically connected in parallel to the first module. Coating evaluation will be made for 1 shot, 5 shot and 10 shot sequences. If the quality of the coating shows a progressive improvement with an increase in the number of discharges than a second module will be added. More sample coatings will be made and evaluated and additional modules added if the coating quality continues to improve. This testing will determine when the process reaches steady state. A maximum of 100 discharges at a $30 \mathrm{~Hz}$ rate is possible.

Arc Initiator. For snowplow to occur, a plasma sheet must be formed at the initiation of current and maintained throughout the current pulse. There are instabilities which can cause the plasma sheet to collapse into a filament. A filament will not expand into a sheet.

To form this plasma sheet, one of the rails has a slot in it with a tungsten electrode centered in the slot. Argon gas is ported into this slot. An RF power supply is connected to the electrode and the rail, with the electrode acting as the cathode.

The argon gas is ionized around the electrode and fills a portion of the railgun's bore. When the PFN discharges into the railgun the region of ionization is extended from one rail to the other, creating a sheet of plasma.

The arc initiator has been tested on a bench test railgun. The details of that testing and a complete description of the arc initiator is presented in a companion paper in these conference proceedings [4].

Powder Feeder. The powder is fed continuously into the last $1 / 3$ of the SBA (the drift zone), using a Metco 4MPDual powder feeder at a rate of $24 \mathrm{~g} / \mathrm{min}$. The powder injection ports are designed to create a powder flow pattern which fills the bore. The injection rate is sufficient to fill the drift region with powder before the next discharge. The region is considered to be filled when the mean distance between powder particles is equal to 10 powder particle diameters. A sufficient distance between particles is needed to make sure that one particle does not shield another from the shocked gas flow field. It may be possible to decrease this distance and thereby increase the material deposition rate. This possibility will be explored during testing.

Control System. The control system consists of:

1) the IGBT firing block

2) the SCR firing block

3) the TVS firing block
4) the sensor block

5) the controller block

Each block is totally isolated with no galvanic connections (when a discharge sequence is in process) to the other blocks or a common ground. Low voltage power requirements are provided with Lithium batteries. Every block has its own motor/alternator power supply for higher power requirements. All communications are transmitted with fiber optics. In this way spurious signals generated by the high electromagnetic fields during the recharge and discharge cycles are avoided. All of the electronics are housed in EMI shielded enclosures.

The control logic is implemented with hardware using voltage comparators. The circuit boards are expandable so that programmable comparators can be installed, allowing fixed control parameters to be downloaded from a computer. This hardware based control system provides microsecond response time without the cost associated with a similarly fast microprocessor based system.

Hypotheses Verification. Two critical hypotheses which will be examined during testing are:

1) A particle traveling at $2 \mathrm{~km} / \mathrm{sec}$ has enough kinetic energy to melt itself upon impact and impart to the immediate substrate sufficient thermal energy to create a fusion bond.

2) Successive discharge impacts are required on the substrate to enhance deposition efficiency.

The first hypothesis comes from energy conversion calculations. It can most readily be confirmed by first achieving the $2 \mathrm{~km} / \mathrm{sec}$ impacts and then evaluating bond quality through metallurgical analysis. Also, high speed photographs of particles striking the substrate and subsequent observations of rebound or melting will allow confirmation of the hypothesis. Melt and subsequent flow on the substrate can also be evaluated.

The second hypothesis is that a single railgun discharge will produce a poor coating with a low depositional efficiency and that many discharges in rapid succession will be necessary to enhance the coating process.

Coating Evaluations. The material combinations which will be explored with the laboratory system are:

1) Inconel 718 on Inconel 718

2) Chrome on a Nickel Alloy

3) and Titanium on Titanium.

The high particle velocities are expected to produce fusion coatings, i.e. a melting across the substrate/coating interface. Scanning electron microscop (SEM) and surface testing can be performed on thin coatings, but a 2 to $3 \mathrm{mil}$ buildup will be needed for metallographic examination. Fifty discharges will be required to achieve a 3 mil coating thickness. This will require five, 10 -shot sequences. Between each sequence the substrate will cool down and this may degrade the coating quality. At this time a decision on whether to increase the total number of discharges by adding recharge bank modules will be made. 


\section{EPD Future Production System}

As the technology matures, the future direction is toward identifying a production system. The research effort is starting to focus on identifying a power supply that can drive the process on a continuous basis. The challenge is to minimize the total energy required per pulse so that upon rep rating the power requirements remain reasonable. Fundamental energy requirements for the system have been outlined with the finite difference model. Quantities required of the process are:

Kinetic energy of the compressed gas and powder: $1.179 \mathrm{~kJ}$

Arc resistive energy

Rail resistive energy

Total

If the source is power matched to the load, then an additional $4.299 \mathrm{~kJ}$ is absorbed by the power supply. The EPD process is rep rated at $30 \mathrm{~Hz}$ to obtain the mass deposition rate required, indicating a process requiring just over 250 $\mathrm{kW}$ of prime power. This is the average power required of the supply. During the railgun pulse, a peak power of 80 MW is required. The challenge is to identify a continuous rated pulsed power source capable of this power output, properly impedance matched to the load. A trade study is scheduled to answer this question. Under consideration are motor alternator charged PFNs and compensated pulsed alternators. The Center for Electromechanics invented the compensated pulsed alternator in the mid 1970s. This is a rotating machine that can be designed to produce the flat topped current pulse required of the process. The pulsed power community in general does not have the experience with producing pulsed power on a continuous basis. The challenge of the technology selected will be the production of a robust power source whose thermal management system is optimized to perform the rep rate requirement.

\section{Conclusions}

An electromagnetic deposition system is underdevelopment which promises to produce coatings superior to those from current state of the art processes, i.e. HVOF and DGun. A particle velocity of $2 \mathrm{~km} / \mathrm{sec}$ should produce fusion bonding. A bench test railgun has been built and tested. Diagnostic measurements of the bench test railgun showed that its performance matched simulation results. A multidischarge laboratory EPD system has been designed and will be tested by August 1997. The next objective will be the design and fabrication of a production system.

\section{Acknowledgment}

This research was sponsored by the United States Air Force, Tinker Air Force Base at Oklahoma City, through a contract administered by ARINC Corporation.

\section{References}

1 R. McCune, "An Exploration of the Cold Gas-Dynamic Spray Method for Several Materials Systems", Proc. of the 8th National Thermal Spray Conf., 1995.

2 J.W. Shearer, "Xenon Shock Waves Driven by High Magnetic Fields", Proc. 2nd International Conf. On Megagauss Magnetic Fields, 1979.

3 J. Uglum, J. Bacon, D. Davis, R. Polizzi, R. Sledge, R. Zowarka "Scaling Analysis of the Electromagnetic Powder Deposition Gun", Proceedings of this conference.

4 R. Sledge, J. Bacon, D. Davis, R. Polizzi, J. Uglum, R. Zowarka, "Arc Initiation for an Electromagnetic Deposition (EPD) Gun", Proceedings of this conference. 